\title{
Dynamic Changes in the Rhizosphere Bacterial Community in Monoculture and Intercropped Corn and Soybean During Various Crop Growth Stages
}

\section{Han Li}

Hunan Academy of Agricultural Sciences

Luyun Luo ( $\square$ luoluyun1992@163.com )

Yangtze Normal University, Chongqin, China https://orcid.org/0000-0003-3513-7229

Bin Tang

Crop Research Institute,Hunan Academy of Agricultural Sciences

Huanle Guo

Crop Research Institute,Hunan Academy of Agricultural Sciences

\section{Zhongyang Cao}

Crop Research Institute,Hunan Academy of Agricultural Sciences

\section{Qiang Zeng}

Crop Research Institute,Hunan Academy of Agricultural Sciences

\section{Songlin Chen}

Crop Research Institute,Hunan Academy of Agricultural Sciences

\section{Zhihui Chen}

Crop Research Institute,Hunan Academy of Agricultural Sciences

\section{Original article}

Keywords: rhizosphere bacterial community, monoculture and intercropping, ACd

Posted Date: August 2nd, 2020

DOI: https://doi.org/10.21203/rs.3.rs-49276/v1

License: (c) (1) This work is licensed under a Creative Commons Attribution 4.0 International License. Read Full License 


\section{Abstract}

Although rhizosphere microorganisms have been studied for a long time, rhizosphere microbial communities based on monoculture and intercropped soybean and corn have rarely been studied. To define the effect of crop monoculture and intercropping on soil physicochemical properties and rhizosphere microbial communities, field experiments were conducted using corn and soybean cultivars at five different crop growth stages. The $\mathrm{pH}$ and soil organic matter (SOM) were the key factors affecting crop rhizosphere soil microorganisms. The content of available phosphorus(AP) in the intercropped soybean/corn soil samples was higher than that in the monoculture soil samples at five different growth stages. The content of available cadmium(ACd) in monoculture soybean rhizosphere soil samples decreased and then increased, but the intercropped soybean soil samples indicated an opposite trend.Proteobacteria, Chloroflexi, Acidobacteria, Actinobacteria, and Firmicutes were the dominant phyla in the soybean and cornrhizosphere soil samples. Crops of the same plant species showed little difference in the bacterial community diversity under the two planting modes. The results showed that the cadmium content in cadmium-polluted soil could be reduced by the soybean/corn intercropping system. The crop rhizosphere bacterial community is not only regulated by the crop itself but also influenced by the planting pattern.

\section{Introduction}

The micro-environment in plant roots that interacts closely with soil is called the rhizosphere and includes many components, such as carbohydrates, amino acids and growth substances (Berendsen et al., 2012). It is a complex dynamic microecosystem in which the physicochemical and biological properties are different from those of the surrounding soil during the processes of plant growth and compound absorption and secretion (Berendsen et al., 2012). Soil microorganisms can affect and change the supply of soil nutrients by producing various organic acids, hormones, antibiotics, alcohols, vitamins and other products (Manching et al., 2014). As an important part of the soil environment, soil microorganisms play an important role in the transformation and increase of soil nutrients and organic matter and thus affect the growth, development and yield and quality of crops (Mendes et al., 2013). The nutrient status of soil can be reflected by the species, number and activity of soil microorganisms (Manching et al., 2014). A healthy rhizosphere environment is an important basis for crop growth. Understanding the influencing factors and diversity of the rhizosphere microbial community has great significance for crop growth and contaminated land remediation.

Many studies have shown that the use of intercropping and crop rotation can change the soil environment by enhancing the ability of plants to make full use of water and nutrients and inhibiting weeds, diseases, and insect pests (Ball et al., 2005; Dai et al., 2009). Intercropping, which means that two or more crops are planted on the same land, of legumes and gramineae crops is an ideal pattern due to interspecific promotion and niche complementation. Gramineae crops require a large amount of nitrogen, which can provide a relatively low nitrogen soil environment for legumes, promoting symbiotic nitrogen fixation by the legumes and improving the nitrogen utilization efficiency of the gramineae crops (Boddey 
et al., 1987). Intercropping of soybean and corn is a common pattern that can improve corn yield (Zaeem et al., 2019). Although there is a competitive relationship between the two crops, soybean yields do not decrease under an appropriate culture environment.

Cd contamination harms the surrounding ecological environment by altering and destroying local ecosystems. Many studies have found that the presence of cadmium will lead to changes in soil properties (Harichová et al., 2012; Hurdebise et al., 2015). Therefore, it is imperative to understand the responses of soil microbial assemblages to cadmium contamination. In our studies, corn (Denghai 605) and soybean (Xiangchun Bean 26) were used as experimental materials. The dynamic changes in the soil physicochemical properties and rhizosphere microbial community of corn and soybean intercropping systems in different growth stages were studied.

\section{Materials And Methods}

\section{Site description and sample collection}

The experiment included two monoculture systems (soybean monoculture and corn monoculture) and an intercropping system (soybean and corn intercropping) in a typical cadmium-polluted fallow zone in Zhuzhou city, Hunan Province, from April to August 2019. Corn (Denghai 605) and soybean (Xiangchundou 26) were used as experimental materials. The physicochemical properties and available cadmium (ACd) content of the rhizosphere soil are shown in Table S1. Each treatment was repeated 4 times, and the plot size was $30 \mathrm{~m}^{2}$. The ratio of corn to soybean rows was 2:3, and the planting density was the same as that used in single cropping. The fertilization amount of corn was $218.25 \mathrm{~kg} / \mathrm{hm} 2$ of nitrogen, $112.5 \mathrm{~kg} / \mathrm{hm}^{2}$ of phosphorus and $112.5 \mathrm{~kg} / \mathrm{hm}^{2}$ of potassium fertilizer. The fertilization amount of soybean was $67.5 \mathrm{~kg} / \mathrm{hm}^{2}$ of nitrogen, $67.5 \mathrm{~kg} / \mathrm{hm}^{2}$ of phosphorus and $67.5 \mathrm{~kg} / \mathrm{hm}^{2}$ of potassium fertilizer, without base fertilizer. The two cultivars were planted in the same experimental field with the same soil properties, climate conditions and field management (such as fertilization and irrigation). Monoculture and intercropped bulk and rhizosphere soil samples were collected in different growth periods, including for the monoculture soybean (D), monoculture corn (Y), intercropped soybean (JD), and intercropped (JY) corn, and were used for determination of the physiochemical properties and classification of rhizosphere microorganisms. Samples were collected at the soybean seedling stage/corn seedling stage (S1), budding stage/jointing stage (S2), pod-bearing stage/tasseling-spinning stage (S3), drum-grain stage/grout stage (S4), and maturity stage/maturity stage (S5), and each sample was randomly collected from five points and mixed into one sample. Bulk soil was considered the surface soil around the plant, and rhizosphere soil was collected by shaking the roots.

\section{Determination Of Soil Physiochemical Properties}

The soil $\mathrm{pH}$ was examined using potentiometry with a $\mathrm{pH}$ meter (PB-10, Sartorious, German). The volumetric method was used to measure the content of soil organic matter (SOM) and available nitrogen 
(AN). UV-Vis spectrophotometry was used to measure the content of available phosphorus (AP). Available potassium (AK) was determined by the inductively coupled plasma-atomic emission spectrometry (ICP-AES).

\section{Dna Extraction, Pcr Amplification And Pyrosequencing}

In total, 80 rhizosphere soil samples were sequenced. Genomic DNA was extracted using a Fast DNA spin kit for soil (MP Biomedicals LLC, USA) according to the manufacturer's instructions. The extracted DNA was stored at $-20^{\circ} \mathrm{C}$ before use. The V3-V4 region of the $16 \mathrm{~S}$ rRNA gene was amplified with primer pairs 338F (5'-ACTCCTACGGGAGGCAGCAG-3') and 806R (5'-GGACTACHVGGGTWTCTAAT-3') (Kong et al., 2019). Both the forward and reverse primers were tagged with unique barcodes to distinguish different samples. The PCR and sequencing processes were performed by Majorbio Bio-Pharm Technology Co. Ltd., Shanghai, China. Sequencing was performed on an Illumina MiSeq platform using a PE250 kit.

\section{Data Analysis And Statistical Analysis}

Data analysis was performed using an open, web-based platform, Galaxy (http://mem.rcees.ac.cn:8080), which comprises an integrated series of bioinformatics tools to aid in intensive bioinformatics research (Kong et al., 2018). Briefly, 12 bp barcode sequences were utilized to sort the different samples. The forward and reverse sequences were then combined with a minimum 30 bp overlap length and a maximum 250 bp maximum overlap length using the FLASH program (Magoc and Salzberg, 2011). Combined sequences with low quality were removed. Subsequently, the reads were clustered into operational taxonomic units (OTUs) at $97 \%$ similarity using UPARSE (Edgar, 2013). The OTU table was resampled with 17,093 sequences to guarantee the same sequencing depth.

The a-diversities (richness, Chao 1, Shannon index, and inverse Simpson index) were calculated, and the relative abundances of phyla and genera were examined in our studies. Weighted principal coordinate analysis (PCoA) based on a weighted UniFrac matrix and dissimilarity tests (nonparametric permutational multivariate (PERMANOVA) based on Bray Curtis) were performed to investigate differences in microbial community structure (Anderson, 2010; Caporaso et al., 2010). The Mantel test was used to evaluate the correlation of physicochemical properties and the microbial community structure. Significance between groups was determined by one-way analysis of variance with SPSS 22 .

\section{Results}

\section{Soil physicochemical properties at different growth stages}

The physicochemical properties of all soil samples collected at 20,40,60,80, and 100 days after the crops were planted at five different stages were measured in succession (Fig. 1). The soil pH of the rhizosphere soil samples from the monoculture soybean all decreased, while that of the corn samples 
increased; intercropped soybean and corn rhizosphere soil samples all decreased and then increased and showed the lowest value at the S3 stage (Fig. 1). The SOM contents in the monoculture and intercropped soybean rhizosphere soil samples all decreased and then increased, and the SOM content of the intercropped corn gradually increased (Fig. 1). The AP, AN, and AK contents in the intercropped soybean rhizosphere soil samples all decreased from the S1 to S3 stage and then rebounded to normal levels at the S3 stage. The AP and AK contents of the monoculture soybean showed no significant changes during the stages, and the AN contents all increased and then decreased; the AN and AK contents of the monoculture corn all decreased and then increased. The AP of the intercropping soybean increased and then rebounded to the normal level at the S3 stage (Fig. 1). The content of ACd in monoculture soybean rhizosphere soil samples all decreased and then increased, but the intercropped soybean soil samples showed the opposite trend. The dynamic trends of the physicochemical properties of the corn rhizosphere soil were opposite to those of the soybean rhizosphere soil (Fig. 1).

\section{Bacterial Community Diversity And Composition At Different Growth Stages}

After a series of steps, a total of 3,881,770 high-quality sequences were obtained from Illumina MiSeq sequencing of 80 rhizosphere soil samples. The observed richness (OTU numbers) and Shannon and inverse Simpson indices of monoculture and intercropped soybean rhizosphere soil samples all decreased and then increased, while monoculture and intercropped corn rhizosphere soil samples showed no significant changes at their different stages (Fig. 2). The Chao1 estimated richness of the monoculture and intercropped corn and soybean rhizosphere soil samples gradually increased at the five stages (Fig. 2). In addition, Student's t-test results between the two groups showed significant differences in the a diversity indices between monoculture corn and soybean at the S2, S3 and S4 stages as well as intercropped corn and soybean at the S1, S2 and S4 stages (Table S2). There were also significant differences in the a diversity indices between monoculture and intercropped soybean at the $\mathrm{S} 4$ stage and monoculture and intercropped corn at the S2 stage (Table S2).

The principal coordinate analysis (PCOA) (Fig. 3) and PERMANOVA results (Table S3) showed that the monoculture soybean rhizosphere bacterial community structure was significantly different from $\mathrm{S} 1$ to S4, while that of the intercropped soybean was significantly different at the S1, S2, S3(S4) and S5 stages. The monoculture and intercropped corn rhizosphere bacterial community structure was significantly different among all five stages $(\mathrm{P}<0.05)$. There was a significant difference in monoculture corn and soybean rhizosphere bacterial community structures among the five stages and in those of intercropped soybean and corn at the S1(S2), S3, S4, and S5 stages. The monoculture and intercropping soybean rhizosphere bacterial community structure was significantly different at the S1, S2 and S4 stages, and the monoculture and intercropped corn rhizosphere bacterial community structure was significantly different at the $\mathrm{S} 2$ and $\mathrm{S} 3$ stages.

Changes in bacterial taxa in rhizosphere soils at five different stages 
The soil bacterial communities were significantly altered during the five different growth stages. The phyla Actinobacteria, Proteobacteria, Chloroflexi, Firmicutes, Acidobacteria, and Bacteroidetes accounted for 88.02 and $96.38 \%$ of those communities in soil and plant samples, respectively (Fig. 4). The relative abundance of the top 25 genera with significant difference in the soil is shown in Table S4. The relative abundance of Sphingomonas and Nocardioides in intercropped soybean in the S1 stage was significantly higher than that of monoculture soybean, and there were no significant differences in the abundance of other species. The relative abundance of Chryseobacterium, and Allorhizobium-NeorhizobiumPararhizobium-Rhizobium in intercropped soybean in the S2 stage was significantly higher than that in monoculture soybean, and the relative abundances of Bradyrhizobium, Intrasporangium, Enterobacter, Acinetobacter, Microbacterium, Uncultured bacterium, Mycobacterium, and Ktedonobacter were significantly lower than those of monoculture soybean. The relative abundance of Bacillus, Fictibacillus and Oryzihumus in intercropped corn was significantly higher than that of monoculture corn. Metagenome, uncultured Acidobacteria bacterium, Burkholderia-Caballeronia-Paraburkholderia, and Bryobacterwere significantly lower in abundance in intercropped than in monoculture corn, while there was no significant difference in other genera. In the S3 period, the relative abundance of Bacillus and Mesorhizobium in intercropped soybean was significantly higher than that of monoculture soybean. Enterobacter and Sphingobacterium showed a significantly lower relative abundance in intercropped than in monoculture soybean. The relative abundance of Streptomyces in intercropped corn was significantly lower than that in monoculture corn, and there was no significant difference in other genera. The relative abundance of Bradyrhizobium in the intercropped soybean samples was significantly higher than that in the monoculture soybean samples in S4. The relative abundances of Intrasporangium, Metagenome, Acinetobacter and Oryzihumus were significantly lower in intercropped soybean than in monoculture soybean, and the relative abundance of Streptomyces was significantly lower than that in monoculture corn, with no other significant differences. During the S5 period, the relative abundance of Enterobacter microbes in intercropped soybean was significantly lower than that in monoculture soybean, while that of Metagenome and Luedemannella was significantly lower than that of monoculture corn, and there were no other significant differences.

\section{The Relationship Between Physicochemical Properties And The Bacterial Community}

The mantel test was performed to examine the correlation between physicochemical properties and bacterial community composition. The results showed that $\mathrm{pH}$ and $\mathrm{SOM}$ were significantly correlated with the rhizosphere soil bacterial communities $(P<0.05$, Table 1$)$. The SOM had the highest correlation with the rhizosphere soil bacterial community (Bray-Curtis distance, $r=0.2756, P=0.001$ ), with no significant correlation found between AP, AN, AK, ACd and the rhizosphere soil microbial community. To determine the relative contribution of environmental variables to the bacterial community, canonical correspondence analysis (CCA) and CCA-based variation partitioning analysis (VPA) were further performed. CCA-based VPA showed that $\mathrm{pH}, \mathrm{SOM}, \mathrm{ACd}$, and available $(\mathrm{P}, \mathrm{K}, \mathrm{N})$ explained $2.82 \%, 3.43 \%$, 
$1.34 \%$ and $5.43 \%$ of the variation in the rhizosphere soil bacterial community, respectively. Their interaction could explain $3.02 \%$ of the variation, leaving $83.96 \%$ of the variation unexplained (Fig. 5).

Table 1

Mantel analysis of the relationship between the microbial

community structure and soil properties. P-values were

calculated using the distribution of the Mantel test statistics

estimated from 9999 permutations. *:P<0.05, **:P<0.01, ***:P $<0.001$.

\begin{tabular}{|lllll|}
\hline Soil properties & \multicolumn{2}{l}{ Bray-Curtis } & \multicolumn{2}{l|}{ Jaccard } \\
\cline { 2 - 5 } & $\mathbf{r}$ & $\mathbf{p}$ & $\mathbf{r}$ & $\mathbf{p}$ \\
\hline pH & 0.1344 & $0.037^{*}$ & 0.1456 & $0.024^{\star}$ \\
\hline SOM & 0.2756 & $0.001^{\star * \star}$ & 0.293 & $0.001^{\star * \star}$ \\
\hline AP & -0.0505 & 0.72 & -0.0271 & 0.585 \\
\hline AN & -0.0366 & 0.699 & -0.0237 & 0.619 \\
\hline AK & -0.0783 & 0.879 & -0.0522 & 0.766 \\
\hline ACd & -0.0319 & 0.573 & -0.0431 & 0.695 \\
\hline
\end{tabular}

\section{Discussion}

Plants release chemicals to the surrounding environment by allelopathy to affect other plants and microbes (Inderjit and Jacob, 2001). Allelopathy produced by secondary substances between different intercropped crops is widespread in nature, and these allelochemicals may also directly or indirectly affect the soil microbial community structure, number, composition and diversity (Inderjit and Jacob, 2001). In our previous study, we found that the corn/soybean intercropping pattern caused differences in cadmium uptake between corn and soybeans, possibly due to alterations in the soil microbial communities In this study, high-throughput sequencing was used to analyze the rhizosphere bacterial communities of corn and soybean crops at five different growth stages, and the dynamic changes in soil physical and chemical properties and ACd contents at five different stages were also analyzed to reveal plant-microbe interactions.

Soil microorganisms participate in many ecological processes in nature and have a great influence on soil quality and function (Rovira, 1965). In our study, we analyzed the contents of $\mathrm{pH}$, organic matter, available $\mathrm{N}, \mathrm{P}, \mathrm{K}$, and available $\mathrm{Cd}$ in the soil of soybean and corn intercropping systems at different growth stages. Available N, P, and K refer to the N, P, and K in the soil that is easily absorbed and utilized by crops. Different crop planting patterns will change the nutrient composition of the soil. Previous studies have shown that intercropping enhances soil carbon and nitrogen (Cong et al., 2015). The AN and AK contents of the monoculture and intercropped soybean/corn soil samples all showed the same trend, showing that the soybean/corn intercropping pattern had little effect on soil available $\mathrm{N}$ and $\mathrm{K}$ in this 
study. The AP content in the intercropped soybean/corn soil samples was higher than that in the monoculture soil samples at five different growth stages, which indicated that the soybean/corn intercropping pattern increased the available $\mathrm{P}$ in the soil. In addition, the soil $\mathrm{pH}$ of the monoculture soybean rhizosphere samples decreased, while that of the corn samples increased, indicating differences in rhizosphere soils among different species and growth stages. The $\mathrm{pH}$ of intercropped soybean and corn rhizosphere soil samples all decreased and then increased, showing the differences in rhizosphere soils at different growth stages (Fig. 1). Previous studies showed that $\mathrm{pH}$ and organic matter were both important factors affecting crop growth and soil microorganisms (Jung et al., 2008; Wang et al., 2019). In our study, the mantel results also indicated that $\mathrm{pH}$ and organic matter were the key factors affecting crop rhizosphere soil microorganisms (Mantel, $\mathrm{P}<0.05$ ).

The content of ACd in monoculture soybean rhizosphere samples all decreased and then increased, but the intercropping soybean soil samples indicated an opposite trend. The dynamic trend of the physicochemical properties of corn rhizosphere soil was opposite to that of soybean rhizosphere soil (Fig. 1). Interestingly, we found that the ACd content in intercropped soybean soil was significantly lower than that in monoculture soybean soil but significantly higher than that in monoculture corn soil compared with that of intercropped corn soil since the S2 stage. With the growth of the two crops, the difference in the ACd content between monoculture and intercropped soybean/corn soil gradually decreased. The results showed that there was a significant difference in the absorption of ACd between intercropping and monoculture planting patterns. The intercropping planting pattern promoted the absorption of ACd in the soybean soil and decreased the absorption of ACd in the corn soil since the S2 period. The results showed that the cadmium content in cadmium-polluted soil could be reduced by the soybean/corn intercropping system (Li et al., 2008).

The dominant phyla in the soybean and corn rhizosphere soil samples were Proteobacteria, Chloroflexi, Acidobacteria, Actinobacteria, Firmicutes, and Firmicutes, which is similar to results reported for other agricultural crops. The main phyla were similar among the rhizosphere of the same crop in single cropping and intercropping systems, but there was a slight difference among the different growth stages. A significant difference in the main phyla was observed between soybean and corn rhizosphere soil samples, which resulted from the crop species genotype (Correa et al., 2010; Sapkota et al., 2015)

When comparing the bacterial community diversity of soybean and corn under the two planting modes, we also found that the same crop showed little difference under the two modes (Yong et al., 2012). However, the bacterial community diversity of corn rhizosphere soil was significantly higher than that of soybean rhizosphere soil. The results showed that corn was enriched with more bacteria than soybean, which reduced the absorption of ACd in corn. Previous research indicates that intercropping planting patterns not only lead to significant changes in microbial diversity but also change the microbial community composition and function (Mei et al., 2009). We found a slight difference in bacterial diversity. In our study, microbial communities of soil samples from different growth stages were significantly different, indicating the significance of crop growth stages in microbial community changes. The bacterial communities of intercropped and monocultured soybean were significantly different at S1, 
S2 and S4, while those of corn were significantly different at the S2 and S3 growth stages. This result indicated that the crop rhizosphere bacterial community is not only regulated by the crop itself but also influenced by the planting pattern. These microorganisms also regulate the rhizosphere environment by crop-microbe interactions (Hu et al., 2020). Reducing the cadmium content in cadmium-contaminated soil through crop-microbial interactions will be our next research focus.

\section{Abbreviations}

SOM

soil organic matter; AP:available phosphorus; ACd:available cadmium; AN:available nitrogen;

AK:Available potassium; pCoA:the principal coordinate analysis; ADONIS:non-parametric permutational multivariate analysis of variance of the Adonis function. OTU:operational taxonomic unit; PERMANOVA:nonparametric permutational multivariate

\section{Declarations}

\section{Acknowledgements}

The authors are thankful to the Crop Research Institute of Hunan Province for their support in this experiment.

\section{Authors' contributions}

$H L$ and $L Y L$ designed the experiments, BY, HL G, ZY C, QZ and SL C performed the experiments, $H L$, and $L Y L$ analyzed the data, $H L$ prepared figures and/or table, $L Y L$ and $Z H C$ revised this manuscript language. All authors read and approved the final manuscript.

\section{Funding}

This work was supported by the National Key Research and Development Program of China \2016YFD0300109; 2016YFD030020907》; the China Agriculture Research System (No.CARS-02); the Natural Science Foundation of Hunan Province邓2018JJ3320》.

\section{Availability of data and materials}

All data obtained have been included into the manuscript and its additional files.

\section{Ethics approval and consent participate}

Not applicable.

\section{Consent for publication}

Not applicable.

\section{Competing interests}

The authors declare that they have no competing interests.

\section{Author details}

1 Crop Research Institute, Hunan Academy of Agricultural Sciences, Changsha, China; 2 Yangtze Normal University, Chongqin, China 


\section{References}

1. Anderson MJ (2010) A new method for non-parametric multivariate analysis of variance. Austral Ecol 26:32-46

2. https://doi.org/10.1111/j.1442-9993.2001.01070.pp.x

3. Ball BC, Bingham I, Rees B, Watson C, Litterick A (2005) The role of crop rotations in determining soil structure and crop growth conditions. Canadian Journal of Soil Science,85(5):557-577

4. $h t t p s: / / 10.4141 / S 04-078$

5. Berendsen RL, Pieterse CM, Bakker PA (2012) The rhizosphere microbiome and plant health. Trends Plant Sci 17(8):478-486

6. https://10.1016/j.tplants.2012.04.001

7. Boddey RM, Knowles R (1987) Methods for quantification of nitrogen fixation associated with Gramineae. Critical Reviews in Plant Sciences, 6(3): 209-266

8. https://doi.org/10.1080/07352688709382251

9. Caporaso JG, Kuczynski J, Stombaugh J, Bittinger K, Bushman FD, Costello EK, Fierer N, Peña AG, Goodrich JK, Gordon JI, Huttley GA, Kelley ST, Knights D, Koenig JE, Ley RE, Lozupone CA, McDonald D, Muegge BD, Pirrung M, Reeder J, Sevinsky JR, Turnbaugh PJ, Walters WA, Widmann J, Yatsunenko T, Zaneveld J, Knight R (2010) QIIME allows analysis of high-throughput community sequencing data. Nat Methods 7:335-336

10. https://doi.org/10.1038/nmeth.f.303

11. Cong WF, Hoffland E, Li L, Six J, Sun JH, Bao XG, Zhang FS, Van Der Werf W (2015) Intercropping enhances soil carbon and nitrogen. Glob Change Biol 21(4):1715-1726

12. https://10.1111/gcb.12738

13. Correa OS, Romero AM, Montecchia MS, Soria MA (2010) Tomato genotype and Azospirillum inoculation modulate the changes in bacterial communities associated with roots and leaves. J Appl Microbiol 102(3):781-786

14. https://10.1111/j.1365-2672.2006.03122.x

15. Dai CC, Xie H, Wang XX, Li PD, Zhang TL, Li YL, Tan X (2009) Intercropping peanut with traditional Chinese medicinal plants improves soil microcosm environment and peanut production in subtropical China. African Journal of Biotechnology 8(16):3739-3746

16. $h$ ttps://10.5897/AJB09.288

17. Edgar RC (2013) UPARSE: highly accurate OTU sequences from microbial amplicon reads. Nat Methods 10:996-998

18. https://doi.org/10.1093/bioinformatics/btr507

19. Harichová J, Karelová E, Pangallo D, Ferianc P(2012) Structure analysis of bacterial community and their heavy-metal resistance determinants in the heavy-metal-contaminated soil sample. Biologia $67,1038-1048$ 
20. https://doi.org/10.2478/s11756-012-0123-9

21. Hu J, Wei Z, Kowalchalchuk G, Xu YC, Shen QR, Jousset A (2020) Rhizosphere microbiome functional diversity and pathogen invasion resistance build up during plant development. Environmental Microbiology, 2020

22. https://10.1111/1462-2920.15097

23. Hurdebise $Q$, Tarayre $C$, Fischer $C$, Colinet G, Hilligsmann S, Delvigne $F(2015)$ Determination of zinc, cadmium and lead bioavailability in contaminated soils at the single-cell level by a combination of whole-cell biosensors and flow cytometry. Sensors 15, 8981-8999

24. https://doi.org/10.3390/s150408981

25. Inderjit, Jacob W(2001) Plant allelochemical interference or soil chemical ecology? Perspectives in Plant Ecology, Evolution and Systematics, 2001.

26. https://10.1078/1433-8319-00011

27. Kong X, Jin DC, Jin SL, Wang ZG, Yin HQ, Xu MY, Deng Y (2018) Responses of bacterial community to dibutyl phthalate pollution in a soil-vegetable ecosystem. Journal of Hazardous Materials 353:5

28. https://10.1016/j.jhazmat.2018.04.015

29. Jung H, Sohn KD, Neppolian B, Choi H (2008) Effect of soil organic matter (SOM) and soil texture on the fatality of indigenous microorganisms in intergrated ozonation and biodegradation. J Hazard Mater 150(3):809-817

30. https://10.1016/j.jhazmat.2007.05.032

31. Kong X, Jin DC, Tai X, Yu H, Duan GL, Yan XL, Pan JG, Song JH, Deng Y (2019) Bioremediation of dibutyl phthalate in a simulated agricultural ecosystem by Gordonia sp. strain QH-11 and the microbial ecological effects in soil. Science of The Total Environment 691-700

32. https://10.1016/j.scitotenv.2019.02.385

33. Li NY, Li ZA, Ding YZ, Zou B, Zhuang P (2008) Effects of intercropping different crops with corn on the Cd uptake by corn. Chin J Appl Ecol 19(6):1369-1373

34. Magoc T, Salzberg SL (2011) FLASH: fast length adjustment of short reads to improve genome assemblies. Bioinformatics 27:2957-2963

35. https://doi.org/10.1093/bioinformatics/btr507

36. Manching HC, Balintkurti PJ, Stapleton AE (2014) Southern leaf blight disease severity is correlated with decreased corn leaf epiphytic bacterial species richness and the phyllosphere bacterial diversity decline is enhanced by nitrogen fertilization. Front Plant Sci 5:403

37. https://doi.org/10.3389/fpls.2014.00403

38. Mendes R, Garbeva P, Raaijmakers JM (2013) The rhizosphere microbiome: significance of plant beneficial, plant pathogenic, and human pathogenic microorganisms. FEMS Microbiol Rev 37:634663

39. https://doi.org/10.1111/1574-6976.12028

Page $11 / 18$ 
40. Rovira AD (1965) Interactions between plant roots and soil microorganisms. Annrevmicrobiol 19(1):241-266

41. https://10.1146/annurev.mi.19.100165.001325

42. Sapkota R, Knorr K, Jorgensen LN, O'Hanlon KA, Nicolaisen M (2015) Host genotype is an important determinant of the cereal phyllosphere mycobiome. New Phytol 207(4):1134-1144

43. https://10.1111/nph.13418

44. Wang CY, Zhou X, Guo D, Zhao JH, Yan L, Feng GZ, Yu GQ, Zhao H L P (2019) Soil pH is the primary factor driving the distribution and function of microorganisms in farmland soils in northeastern China. Ann Microbiol 69(13):1461-1473

45. https://10.1007/s13213-019-01529-9

46. Yong TW, Yang WY, Xiang DB, Zhu ZY (2012) Effect of Wheat/Corn/Soybean and Wheat/Corn/Sweet Potato Relay Strip Intercropping on Bacterial Community Diversity of Rhizosphere Soil and Ni-trogen Uptake of Crops. Acta Agronomica Sinica 38(2):333-343

47. https://10.3724/SP.J.1006.2012.00333

48. Zaeem M, Nadeem M, Pham TH, Ashiq W, Thomas R (2019) The potential of corn-soybean intercropping to improve the soil health status and biomass production in cool climate boreal ecosystems. Scientific Reports, 9(7)

49. https://10.1038/s41598-019-49558-3

50. Sun YM, Zhang NN, Wang ET, Yuan HL, Yang JS, Chen WX (2009) Influence of intercropping and intercropping plus rhizobial inoculation on microbial activity and community composition in rhizosphere of alfalfa (Medicago sativa L.) and Siberian wild rye (Elymus sibiricus L.). Fems Microbiology Ecology 70(2):62-70

51. https://10.1111/j.1574-6941.2009.00752.x

\section{Figures}


$\mathrm{pH}$

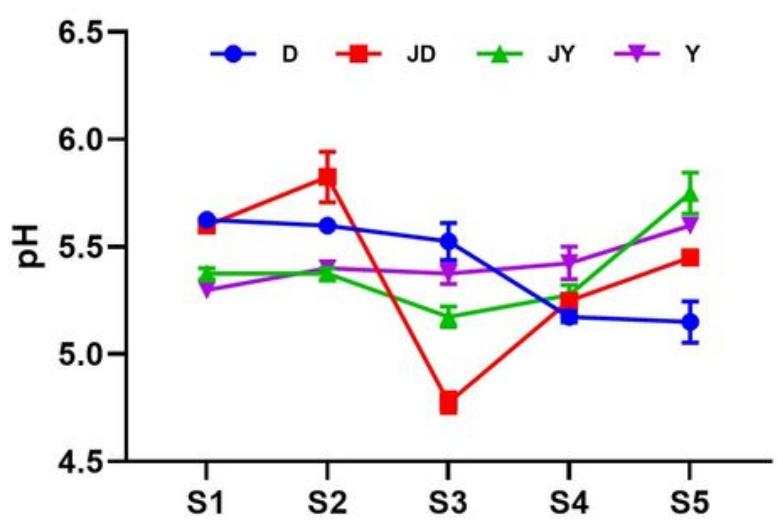

AP

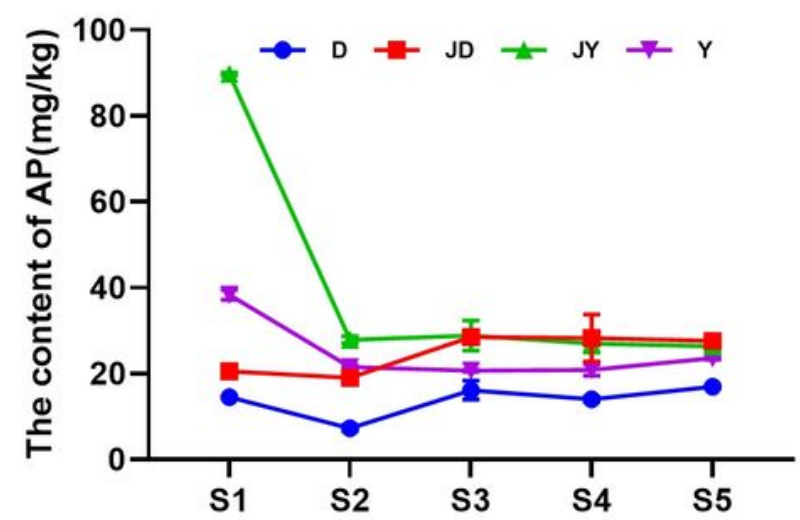

AK

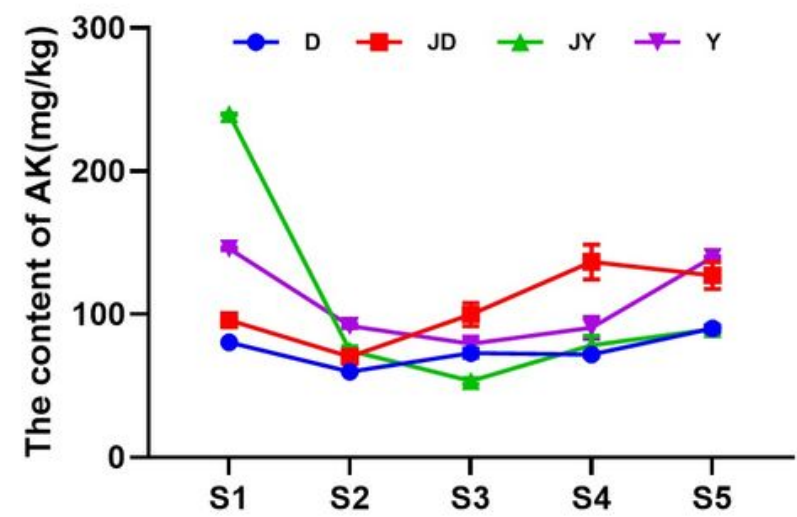

SOM

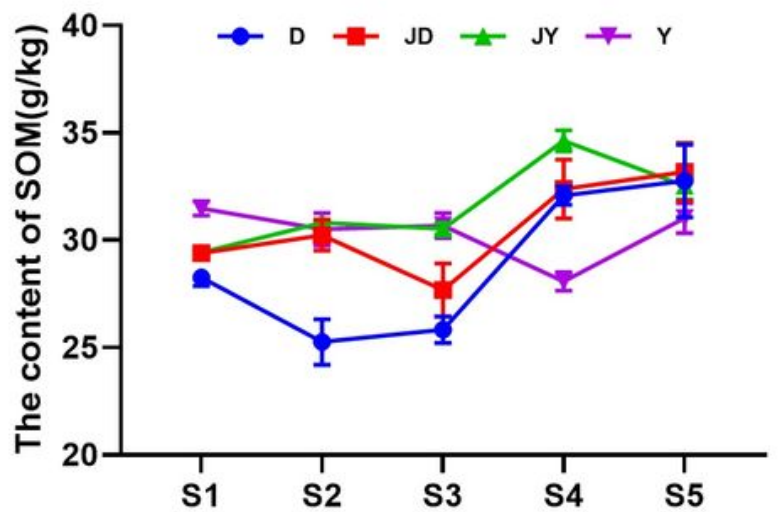

AN

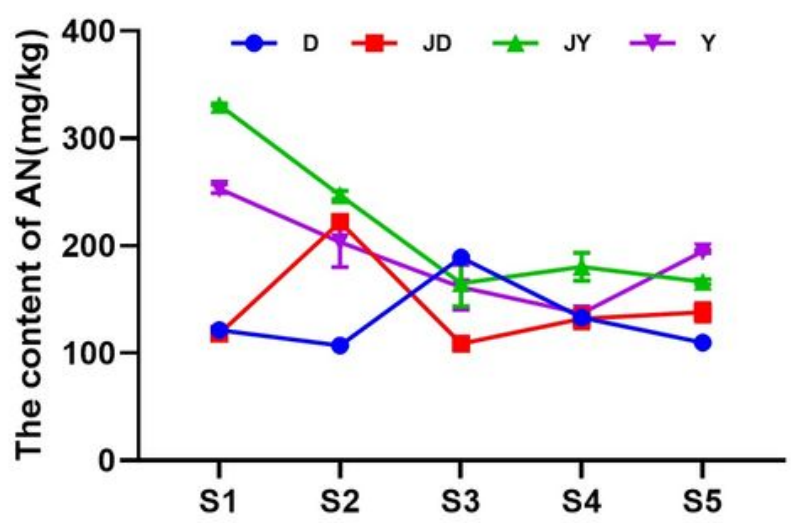

ACd

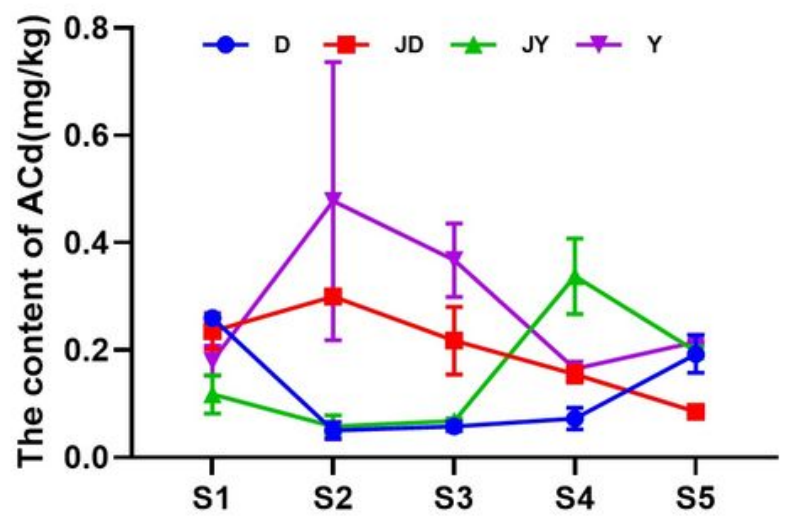

\section{Figure 1}

Physicochemical properties of different rhizo sphere soils. SOM:Soil organic matter, AP: available P,AN:availableN, AK: availableK,ACdavailable Cd.D: the monoculture soybean, Y: monoculture corn, JD:intercropped soybean,JY: intercropped corn. S1: the soybean seedling stage/corn seedling stage, S2: budding stage/jointing stage, S3: pod-bearing stage/tasseling-spinning stage, S4: drum-grain stage/grout stage,S5: maturity stage/maturity stage. 
Shannon

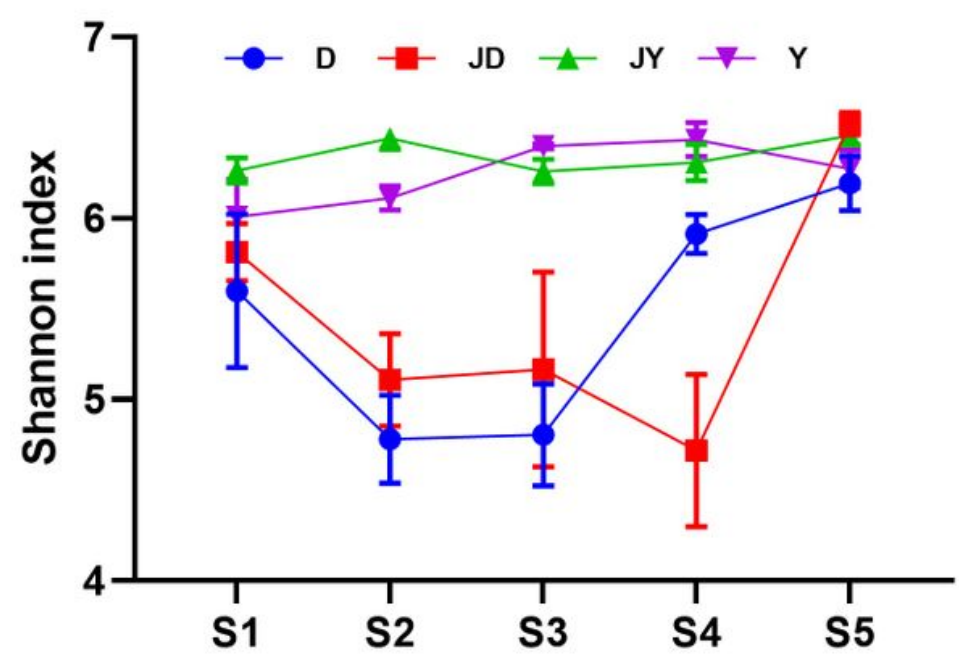

Observed_richness

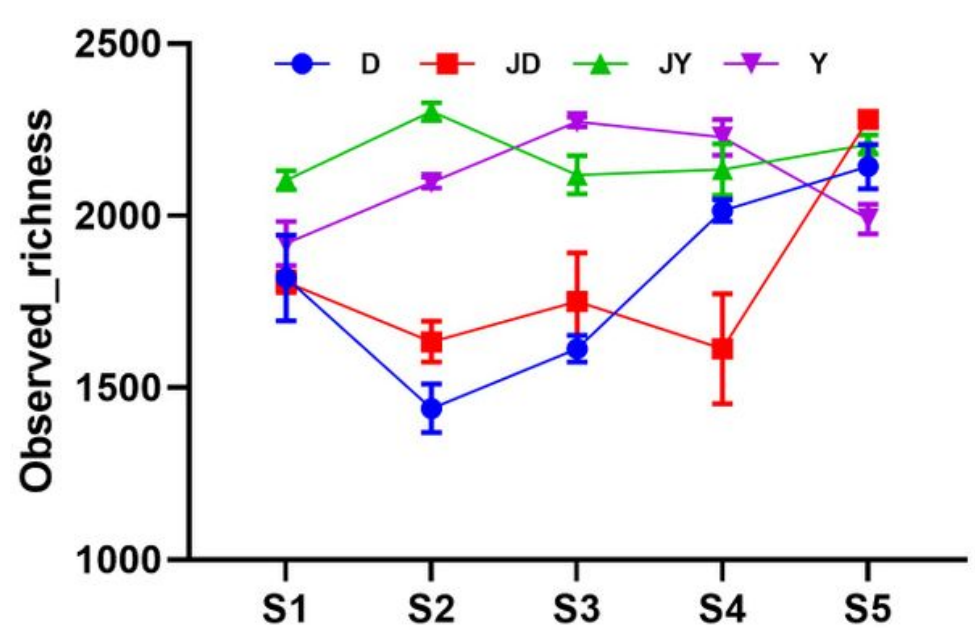

Inv_Simpson

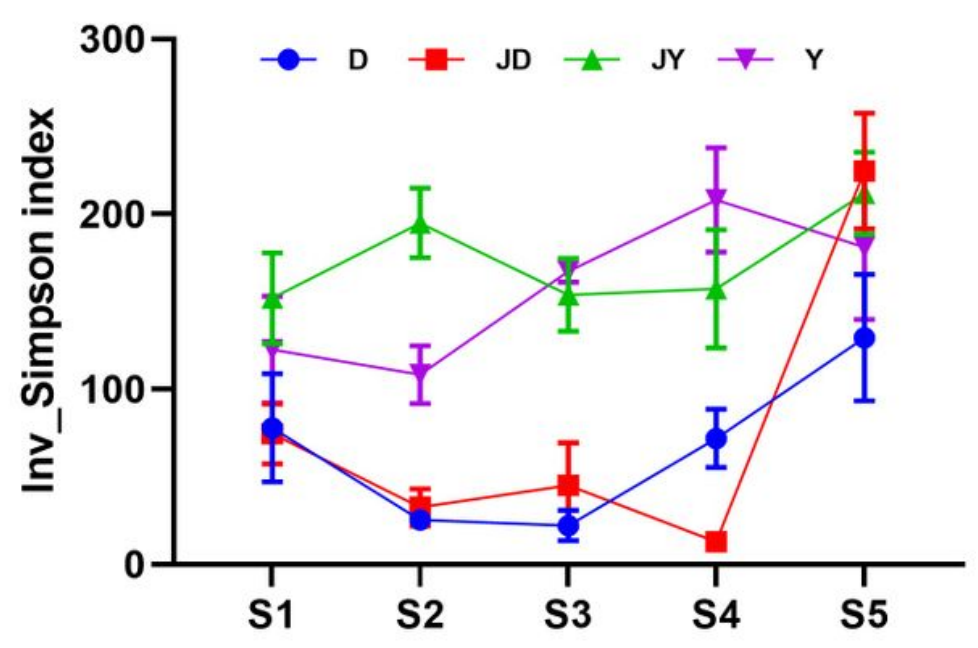

Chao1

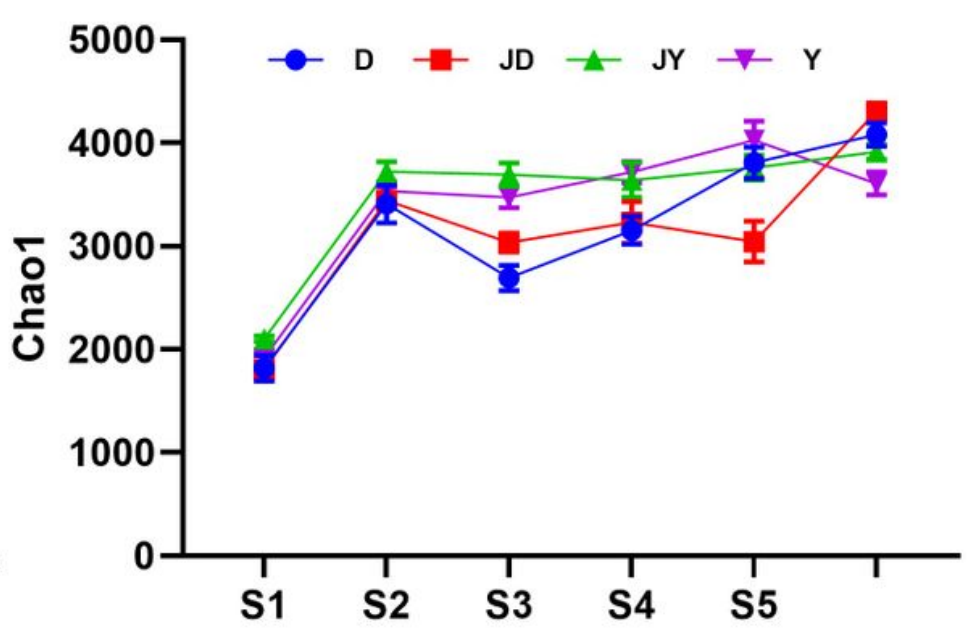

Figure 2

The a diversity of different rhizospheresoils. D: the monoculture soybean, Y: monoculture corn, JD: intercropped soybean,JY: intercropped corn. S1: the soybean seedling stage/corn seedling stage, S2: budding stage/jointing stage, S3: pod-bearing stage/tasseling-spinning stage, S4: drum-grain stage/grout stage,S5: maturity stage/maturity stage. 


\section{weighted}

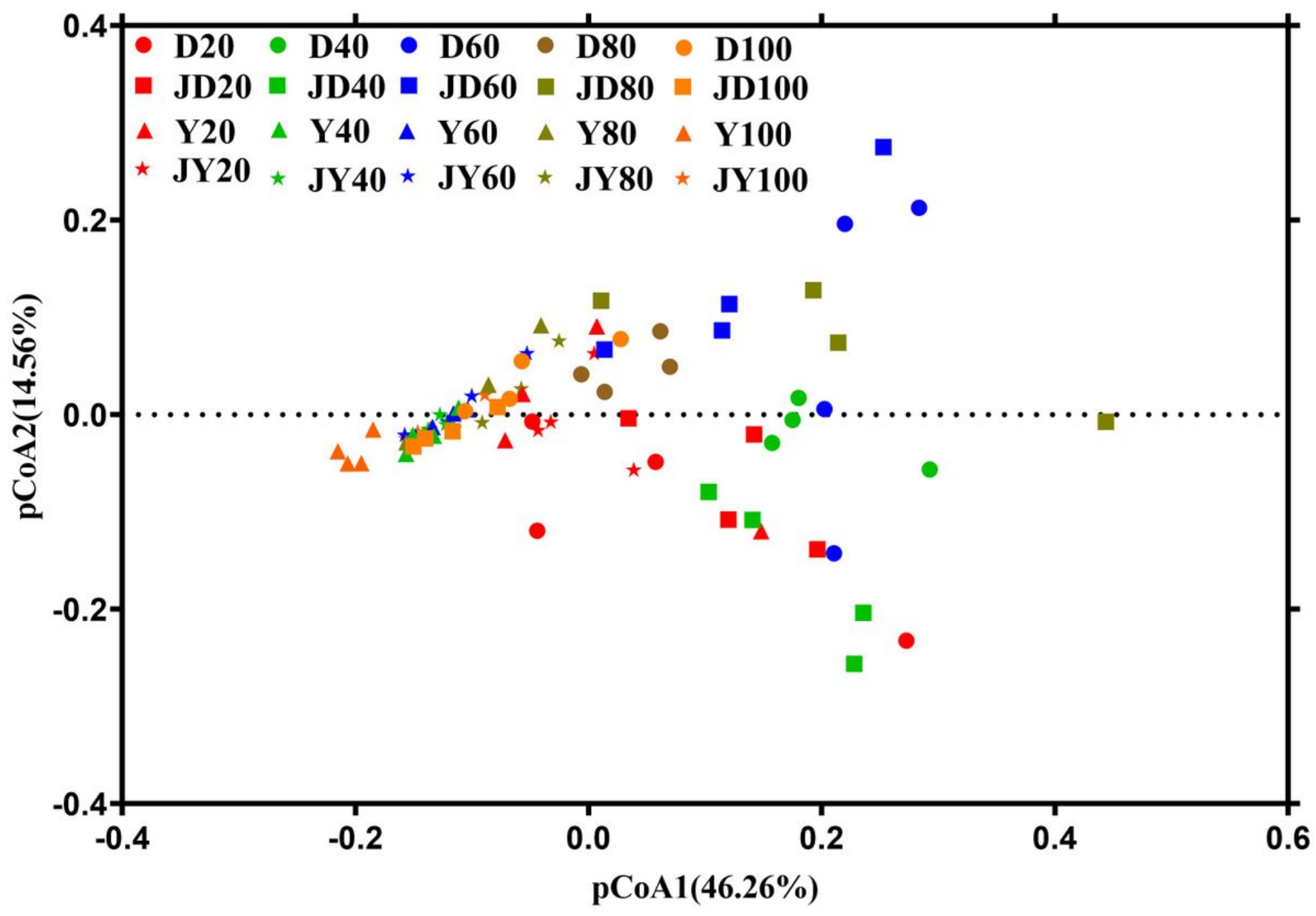

Figure 3

Principal coordinate analysis (weighted_PCoA) of microbial communities based on Bray-Curtis dissimilarity matrices. 


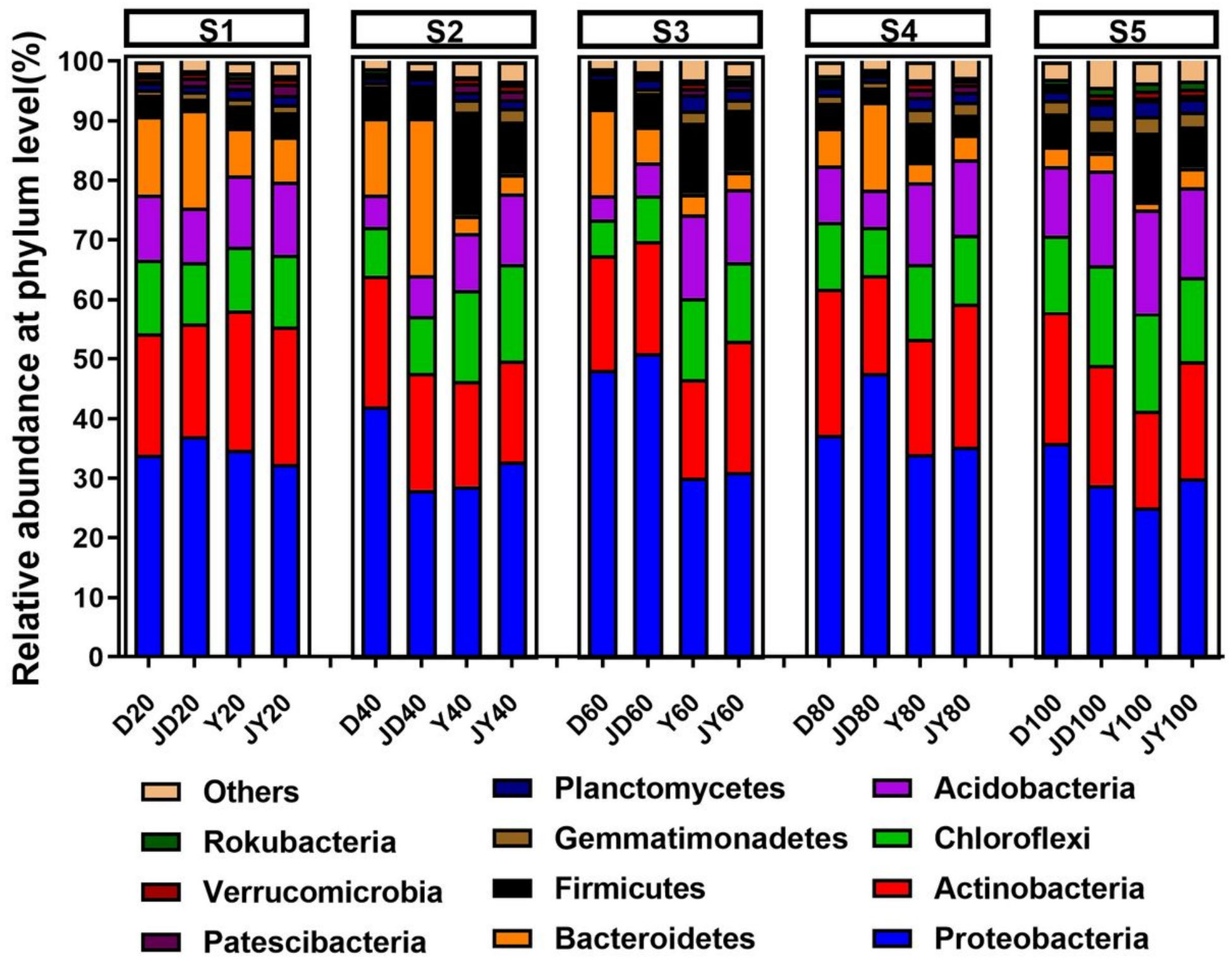

Figure 4

The relative abundance of the dominant phyla in the different rhizospheresoils.D: the monoculture soybean, Y: monoculture corn, JD: intercropped soybean,JY: intercropped corn. S1: the soybean seedling stage/corn seedling stage, S2: budding stage/jointing stage, S3: pod-bearing stage/tasseling-spinning stage, S4: drum-grain stage/grout stage,S5: maturity stage/maturity stage. 
2nd group

3rd group

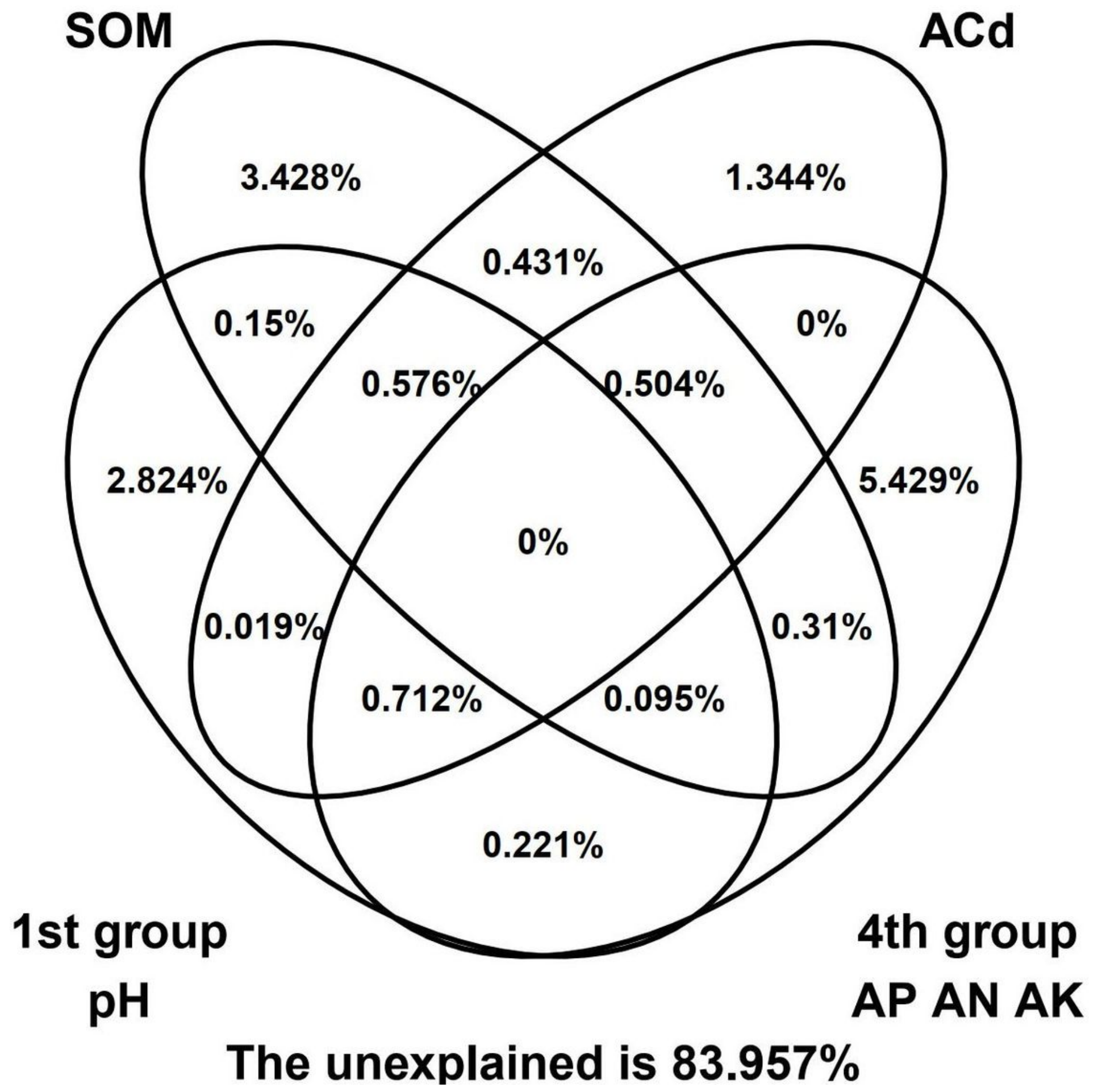

Figure 5

Figure 5

Supplementary Files

This is a list of supplementary files associated with this preprint. Click to download. 
- SupplementTables.docx

Page 18/18 